\title{
An Hourglass truss lattice structure and its mechanical performances
}

\author{
Li-Jia Feng, Lin-Zhi Wu*, Guo-Cai Yu.
}

Center for Composite Materials, Harbin Institute of Technology, Harbin 150001, PR China

\begin{abstract}
This paper focuses on improving the strength of truss lattice sandwich structures by optimizing the topology structure. A new Hourglass truss lattice sandwich structure is then designed and fabricated by a snap-fit method. The out-of-plane and in-plane compressive properties of the Hourglass lattice sandwich structure are investigated theoretically and experimentally, and the measured results are compared with the analytical predictions. Results show that the low relative density Hourglass lattices have higher peak out-of-plane compressive strengths than the pyramidal lattices. Moreover, the peak in-plane compressive loads of Hourglass lattice sandwich structures are superior to the pyramidal sandwich structures. Therefore, the Hourglass lattice sandwich structure can be considered as a promising candidate in high specific strength lightweight structures.
\end{abstract}

Keywords: Hourglass; Truss lattice; Snap-fit method; Compressive properties.

\footnotetext{
* Corresponding author.

Tel.: +86-451-86402549; fax: +86 45186402386 .

E-mail: wlz@hit.edu.cn (Lin-Zhi Wu).
} 


\section{Introduction}

Over the past few years, a variety of truss lattice sandwich structures [1] have been produced with a typical aim of developing high specific strength lightweight structures for the multifunctional application. Several open cell topologies have been proposed based on truss lattices with pyramidal [2-4], tetrahedral [5], 3D-Kagome [6-8] and other topologies [9-12]. Two typical topologies are pyramidal [2,13] and tetrahedral [5,13-14], and they exhibit superior structural characteristics[10,15-18]. Nevertheless, research results show that the strengths of these lattice sandwich structures depend upon the compressive strength of the core and the cell size (which controls the inter-node spacing) [19-20]. Thus, while pyramidal and tetrahedral topology systems usually offer significantly superior structural performance, improvements appear feasible, based on the following two limitations: the lattice cores are susceptible to buckling under compressive loads, and thick core leads to large inter-node spacing which weakens resistance to local face sheet buckling [18,21].

In order to improve the compressive strength of the core, a 3D-Kagome structure is developed [6], providing superior resistance to buckling of the core truss. However, the investment casting fabrication approach can be costly to implement and cast metals are subject to strength knockdown by casting factors. Also, many of the alloys do not favorably respond to heat treatment.

On the other hand, in order to decrease the inter-node spacing, a multi-layer pyramidal lattice sandwich structure is proposed by Cote et al. [15]. Results show that, for a given core thickness, the resistance to local face sheet buckling increases with the number of pyramidal core layers, due to the decrease of the inter-node spacing. However, for a given relative density, the multi-layer pyramidal lattice has the same truss slenderness ratio as the single layer one. Therefore, the resistance to truss buckling of the multi-layer pyramidal lattice is not superior to the single layer one. 
Here, a new truss lattice structure is proposed, called the Hourglass truss lattice. This lattice sandwich structure is envisioned to have all of the three superior structural characteristics at the same time.

(i) Superior resistance to buckling of the core truss: Compared with pyramidal lattice structures, the trusses in the Hourglass lattice have a smaller slenderness ratio, although the relative density is the same.

(ii) Superior resistance to local buckling of the face sheets: For a given core thickness, the inter-node spacing of the Hourglass lattice sandwich structure is the same as a two-layer pyramidal sandwich structure, and is about twice that of a single layer pyramidal sandwich structure.

(iii) Simple and low-defect fabrication method: It can be manufactured by a WEDM and snap-fit approach [12,22-25], which is simple and low-defect.

In this study, an experimental and analytical investigation is carried out to examine the out-of-plane and in-plane compressive strengths of Hourglass truss lattice sandwich structures.

\section{Fabrication approach}

\subsection{Lattice fabrication}

The Hourglass truss lattice has a structure similar to the two-layer pyramidal truss lattice, as seen in Fig. 1. A transition process can be used to illustrate the difference between the two topologies: combining those cells providing no contribution to the decrease of inter-node spacing $d$ (the brown cells in Fig. 1(b)) into other cells (the blue cells in Fig. 1(b)). This process turns four two-layer pyramidal truss unit cells into two Hourglass truss cells (see Fig. 1(c)). Namely, the Hourglass truss cells are arranged more sparsely, and this causes a smaller truss slenderness ratio ( $l / t$ ratio for a strut of length $l$ and square cross section of side length $t$ ) due to the unchanged relative density. Meanwhile, the inter-node spacing $d$ of the Hourglass lattice sandwich structure 
remains unchanged and still twice that of a single layer pyramidal truss lattice (compare the bottoms of Fig. 1(a) to (c)).

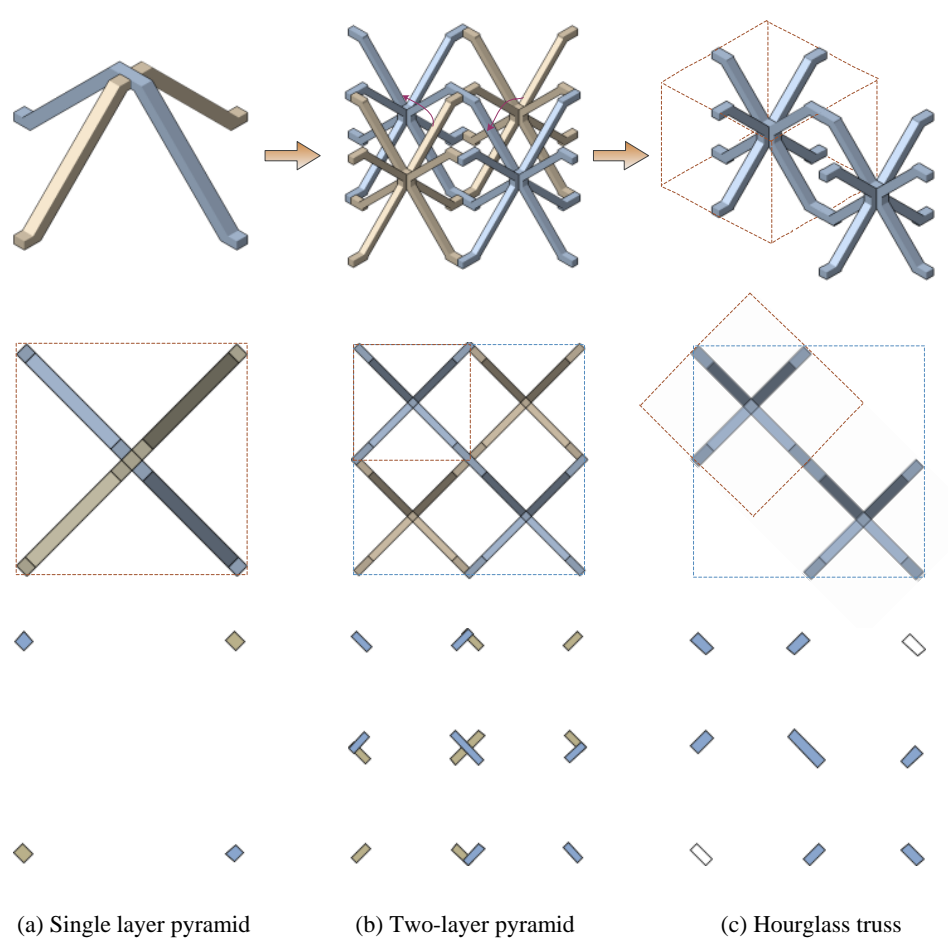

Fig. 1. The process of transformation from a single layer pyramidal truss lattice to an Hourglass truss lattice: (a) Single layer pyramid, (b) Two-layer pyramid and (c) Hourglass truss.

The Hourglass truss lattice sandwich structures are fabricated from 304 stainless steel sheets of a single thickness, $t=1.40 \mathrm{~mm}$, in a three step process summarized schematically in Fig. 2 . The truss row patterns (see Fig. 2(a)) are cut by Wire Electrical Discharge Machining (WEDM) from flat 304 stainless steel sheets. Small slots are cut to ensure the cutting continuity as seen in Fig. 2(a). These patterns are then snap-fitted into each other to form the Hourglass truss lattice, Fig. 2(b). The snap-fit lattice is bonded using a vacuum brazing approach. A Ni-7Gr-4.5Si-3.1B-3Fe amorphous solder alloy (Nicrobraze 31) is first applied to the nodal regions of the assembled structure. The core is then placed between two 304 stainless steel face sheets, a light load is applied to ensure contact. The assembly is placed in a vacuum furnace for high-temperature brazing and heated at $15^{\circ} \mathrm{C} / \mathrm{min}$ up to $950^{\circ} \mathrm{C}$, held for $30-60$ min (to provide a uniform temperature filed in the specimens), then heated at $20^{\circ} \mathrm{C} / \mathrm{min}$ to $1050^{\circ} \mathrm{C}$, for $6-10 \mathrm{~min}$ at $2 \times 10^{-2} \mathrm{~Pa}$ before the furnace naturally cools to ambient temperature. The full Hourglass lattice sandwich structure is shown in Fig. 2(d). A similar 
process is utilized to manufacture the single layer pyramidal lattice sandwich structures(“pyramidal lattice sandwich structures" for short later), and the snap-fit process is seen in Fig. 2(e). The two lattice sandwich structure photographs taken from ones of the brazed lattice structures are shown in Fig. 3.

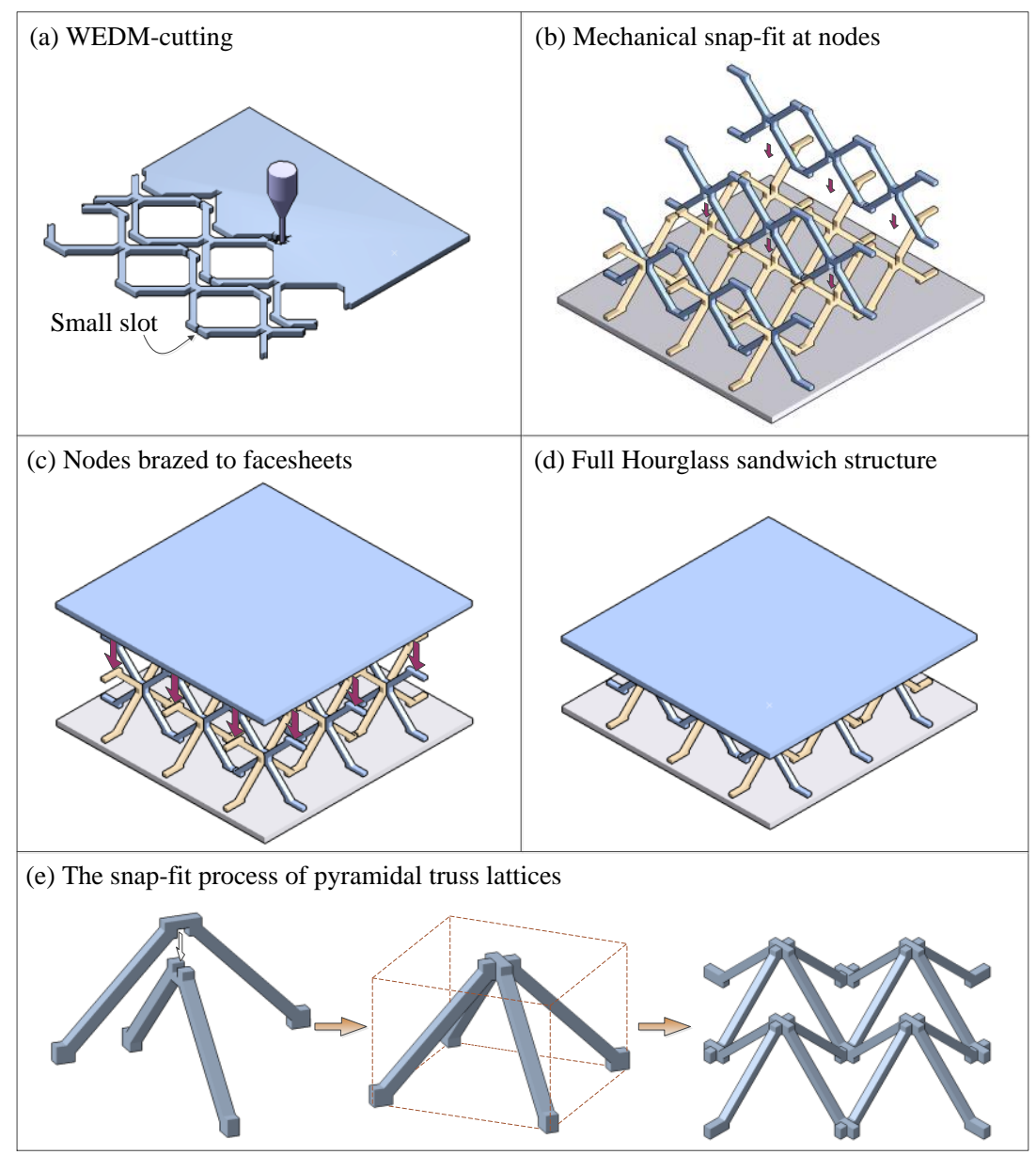

Fig. 2. Schematic illustration of the "snap-fit" lattice fabrication and assembly method for making the Hourglass truss lattice sandwich structure: (a) to (d); The snap-fit process of the pyramidal truss lattice is shown in (e).
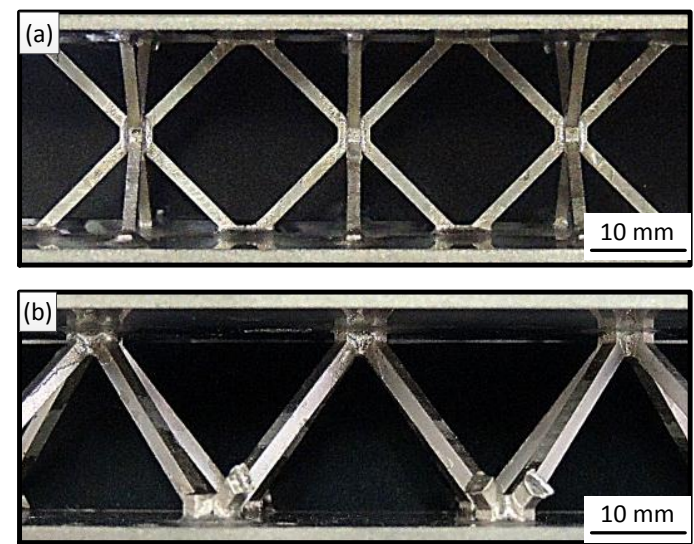

Fig. 3. Photographs of the brazed (a) Hourglass lattice sandwich structure and (b) pyramidal lattice sandwich structure. 


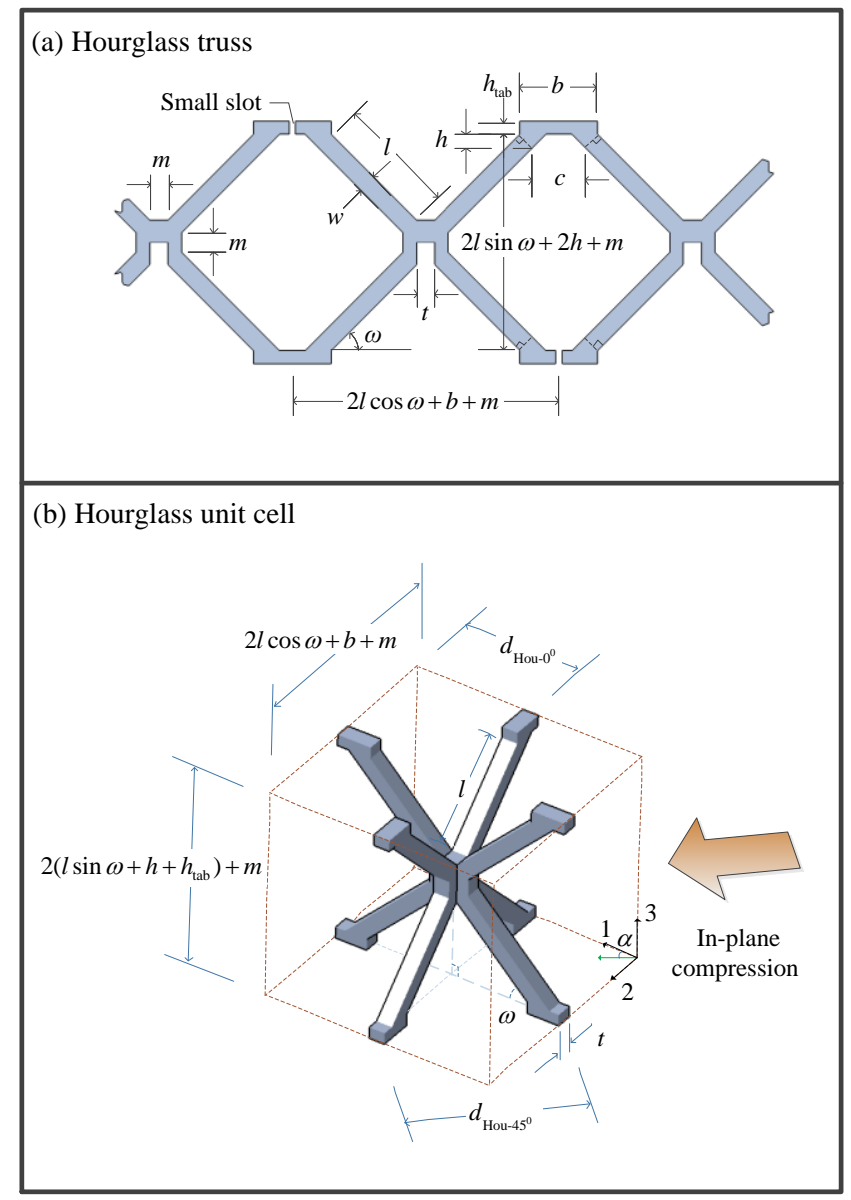

(c) Pyramidal truss

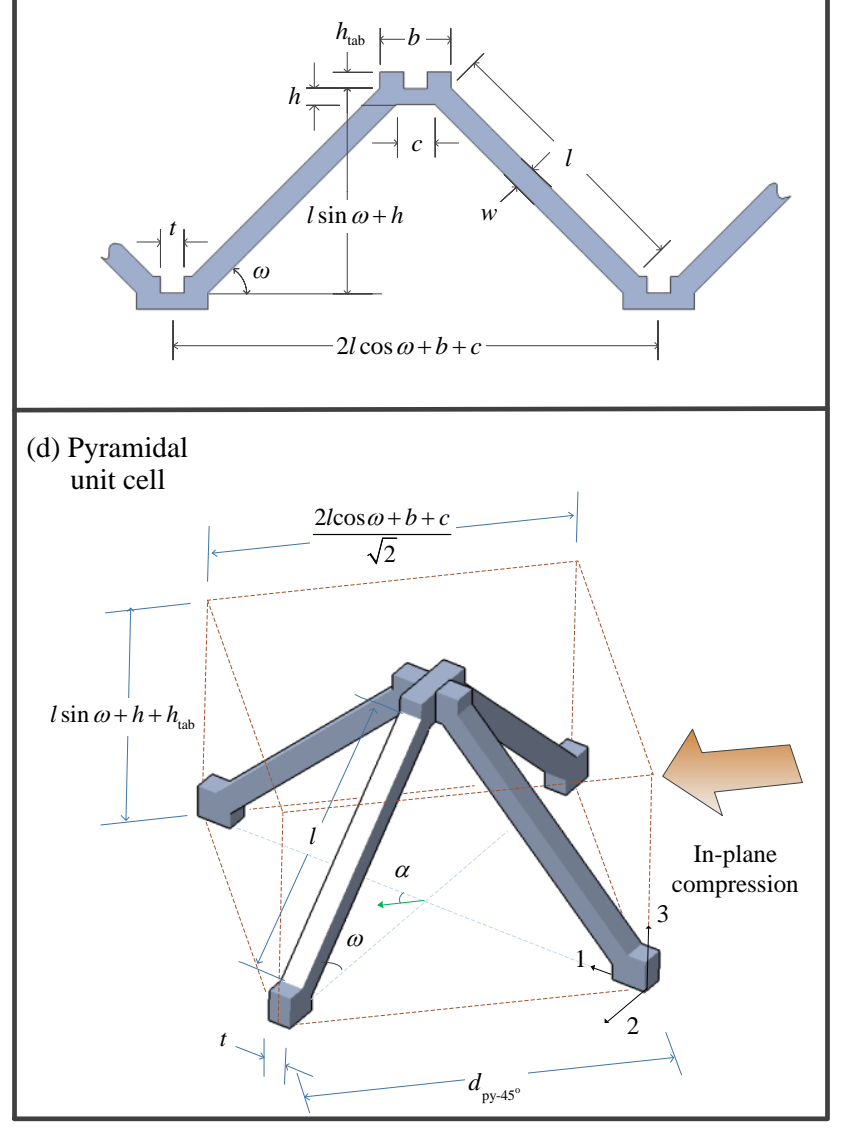

Fig. 4. The geometries with relevant design variables identified of the out-of-plane trusses and the unit cell of the snap-fit Hourglass truss lattice and the pyramidal truss lattice. The Cartesian co-ordinate system and loading directions are also specified.

Consider the 3D unit cells indicated in Fig. 4. Geometrical parameters of the Hourglass truss lattice and the pyramidal truss lattice are defined together with coordinate systems used later. The truss member side length is equal to the side width, $w=t$. Their values are summarized in Table 1 . The arrow indicates the experimental in-plane compressive loading orientation. By calculating the volumes of regions occupied by materials, and scaling this by the unit cell volume, the relative density, $\bar{\rho}$, of the snap-fit Hourglass truss lattice is given by

$$
\bar{\rho}=\frac{2 \sqrt{2} l t^{2}+\left(\sqrt{2}\left(b h_{\mathrm{tab}}+2 h^{2}+\frac{m^{2}}{2}\right)+\frac{3 \sqrt{2}}{4} m t+\frac{1}{2}(1-\sqrt{2}) t^{2}\right) t}{\left(l+\frac{\sqrt{2}}{2}\left(m+2 h+2 h_{\mathrm{tab}}\right)\right) \cdot\left(l+\frac{\sqrt{2}}{2}(b+m)\right)^{2}}
$$

For the snap-fit pyramidal truss lattice 


$$
\bar{\rho}=\frac{4 \sqrt{2} l t^{2}+\left(4 \sqrt{2} b h_{\mathrm{tab}}+2 \sqrt{2}(b+c) h-2 \sqrt{2}\left(h+h_{\mathrm{tab}}\right) \cdot t\right) t}{\left(l+\sqrt{2}\left(h+2 h_{\mathrm{tab}}\right)\right)\left(l+\frac{\sqrt{2}}{2}(b+c)\right)^{2}} .
$$

Eqs. (1) and (2) are arranged in this way to separate volume contributions from the trusses (first term of the numerator) and nodes. It can be shown that in the limit of vanishing node size, Eq. (1) reduces, in a first order approximation, to the relative density expression for the ideal Hourglass truss lattice

$$
\bar{\rho}=2 \sqrt{2}(t / l)^{2}
$$

For the ideal pyramidal truss lattice, Eq. (2) reduces to

$$
\bar{\rho}=4 \sqrt{2}(t / l)^{2}
$$

It is noted that the $(t / l)^{2}$ ratio of the ideal Hourglass truss lattice is twice that of the ideal pyramidal truss lattice when their relative densities are the same.

Table 1

Node and strut dimensions for the Hourglass truss and pyramidal truss lattices used in this study (unit: mm).

\begin{tabular}{lllllllll}
\hline Geometry parameters & $t$ & $w$ & $b$ & $c$ & $h_{\text {tab }}$ & $h$ & $\omega$ & $m$ \\
\hline Hourglass truss & 1.400 & 1.400 & 5.460 & 3.480 & 0.990 & 0.990 & $45^{\circ}$ & 1.500 \\
Pyramidal truss & 1.790 & 1.790 & 5.046 & 2.500 & 1.273 & 1.273 & $45^{\circ}$ & - \\
\hline
\end{tabular}

\subsection{Tensile and compressive response of material}

In order to determine the parent alloy properties and later predict the lattice mechanical properties, traditional tensile test specimens accompany the sandwich samples through the brazing cycle and are measured. However, the trusses under out-of-plane compression and the face sheets under in-plane compression are both subjected to compressive stresses. Thus, the uniaxial compressive response of solid material underwent the same brazing cycle is also measured. Tensile tests are performed according to ASTM E8-01, and compressive tests according to ASTM E9-89a. Three repeated tests are conducted to gauge the variability in each case. 


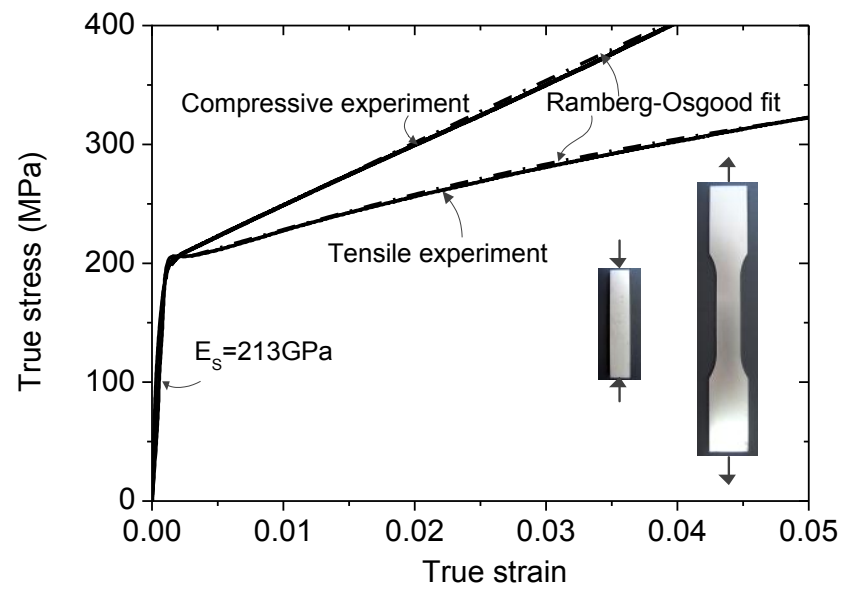

Fig. 5. The tensile and the compressive true stress-strain curve of the 304 stainless steel after exposure to the thermal cycle used for brazing. The modified Ramberg-Osgood fitting curves to both the tensile and compressive stress-strain responses are also shown.

Table 2

Summary of modified Ramberg-Osgood fitting parameters of the tensile and compressive models.

\begin{tabular}{ccccccccc}
\hline & $\begin{array}{c}\sigma_{0.2} \\
(\mathrm{MPa})\end{array}$ & $\begin{array}{c}E_{0} \\
(\mathrm{GPa})\end{array}$ & $n$ & $\begin{array}{c}E_{0.2} \\
(\mathrm{GPa})\end{array}$ & $\varepsilon_{0.2}$ & $m$ & $\varepsilon_{\mathrm{u}}$ & $\sigma_{\mathrm{u}}$ \\
\hline Compressive curve fit & 212 & 213 & 19.63 & 5.27 & 0.003 & 2.74 & 0.044 & 426.46 \\
\hline Tensile curve fit & 206 & 213 & 31.84 & 3.19 & 0.003 & 3.25 & 0.0488 & 320.36 \\
\hline
\end{tabular}

Both the tensile and compressive stress-strain responses are shown in Fig. 5. From the curves,

the elastic properties of the material such as the elastic modulus $E_{\mathrm{s}}$ and $0.2 \%$ offset yield stress $\sigma_{\mathrm{y}}$ are obtained. Meanwhile, the modified Ramberg-Osgood model [26], which uses two equations in Eq. 5, is applied to fit the full range stress-strain curves. Table 2 summarizes the modified Ramberg-Osgood fitting parameters of the tensile and compressive models, where $E_{0}=E_{\mathrm{s}}$ and $\sigma_{0.2}=\sigma_{\mathrm{y}}$. The tangent modulus, $E_{\mathrm{t}}$, is obtained by differentiation of each fitted curve. Then the inelastic bifurcation stress of the compressive facesheet or the compressive strut is obtained using the tangent modulus theory [27-28] as discussed in Section 3.2. Note that the tangent modulus, $E_{\mathrm{t}}$ of the compressive response is clearly higher than that of the traditional tensile model. Thus, the two response predictions will be compared with measurements later, respectively, and the response prediction that agree well with measurements will be selected. 


$$
\varepsilon=\left\{\begin{array}{l}
\frac{\sigma}{E_{0}}+0.002\left(\frac{\sigma}{\sigma_{0.2}}\right)^{n} \quad \text { for } \sigma \leq \sigma_{0.2} \\
\frac{\sigma-\sigma_{0.2}}{E_{0.2}}+\left(\varepsilon_{u}-\varepsilon_{0.2}-\frac{\sigma_{u}-\sigma_{0.2}}{E_{0.2}}\right)\left(\frac{\sigma-\sigma_{0.2}}{\sigma_{u}-\sigma_{0.2}}\right)^{m}+\varepsilon_{0.2} \quad \text { for } \sigma>\sigma_{0.2}
\end{array}\right.
$$

\section{Out-of-plane compression}

In this section, we investigate the effects of the relative density on the out-of-plane compressive behavior of Hourglass truss lattices. The relative density of the truss lattice is controlled by varying the strut length $l$ while keeping all the other geometric parameters fixed. The Hourglass lattice structures along with the pyramidal lattice structures used for comparison are tested in out-of-plane compression at a nominal strain rate of $5 \times 10^{-4} \mathrm{~s}^{-1}$. Three repeated tests are conducted to gauge the variability in each case. Table 3 lists the structure dimensions of each design.

Table 3

Summary of the predictive and experimental values of the out-of-plane compressive strengths.

\begin{tabular}{|c|c|c|c|c|c|c|}
\hline \multirow{2}{*}{ Core type } & \multicolumn{3}{|c|}{ Hourglass truss lattice } & \multicolumn{3}{|c|}{ Pyramidal truss lattice } \\
\hline & $\bar{A}$ & $\mathrm{~B}$ & $\mathrm{C}$ & $\overline{\mathrm{D}}$ & $\mathrm{E}$ & $\mathrm{F}$ \\
\hline$t / l$ & 0.078 & 0.113 & 0.141 & 0.049 & 0.070 & 0.087 \\
\hline Core height $\left(H_{\mathrm{c}}\right)(\mathrm{mm})$ & 30 & 22 & 18.5 & 30 & 22 & 18.5 \\
\hline Relative density $(\bar{\rho})(\%)$ & 1.15 & 1.97 & 2.71 & 0.96 & 1.91 & 2.64 \\
\hline Predicted $\sigma_{33}^{\mathrm{pk}}(\mathrm{MPa})$ & 1.21 & 2.52 & 5.53 & 1.00 & 2.14 & 3.10 \\
\hline Average of the measured $\sigma_{33}^{\mathrm{pk}}(\mathrm{MPa})$ & 1.16 & 2.69 & 4.48 & 0.79 & 2.13 & 3.28 \\
\hline$\%$ error & $-4.11 \%$ & $6.71 \%$ & $-18.93 \%$ & $-20.96 \%$ & $-0.24 \%$ & $5.95 \%$ \\
\hline
\end{tabular}

\subsection{Out-of-plane compression response}

The out-of-plane compressive stress-strain responses for Hourglass truss lattices are summarized in Fig. 6. Hourglass truss lattices exhibit typical characteristics of many cellular metal structures. Following a region of nominally elastic response, gradual core yielding occurs followed by plastic strain hardening to the peak strength. Continued loading results in some "softening" to a plateau region and finally hardening associated with core densification (at a strain of 0.5 0.6) where upon the core exhibited greatly increased load resistance. Fig. 6 reveals that the degree of softening 
depends on the relative density of the truss lattice. The lattice structures with the relative density of $1.15 \%$ inelastic buckle shortly after yielding (at a strain of $\sim 0.02$ ), and the significant core softening is observed after the onset of inelastic buckling.

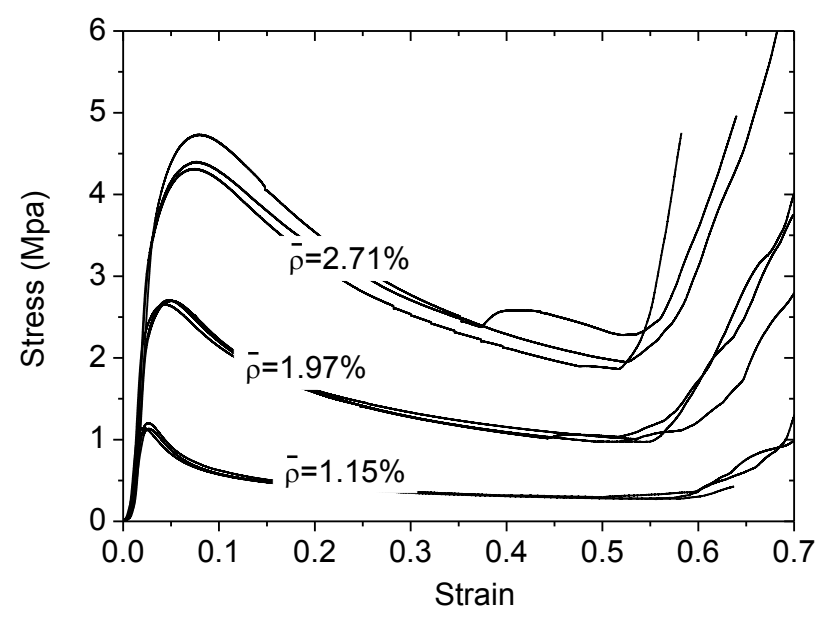

Fig. 6. Out-of-plane compressive stress-strain responses of the snap-fit Hourglass truss lattice samples with different relative densities.

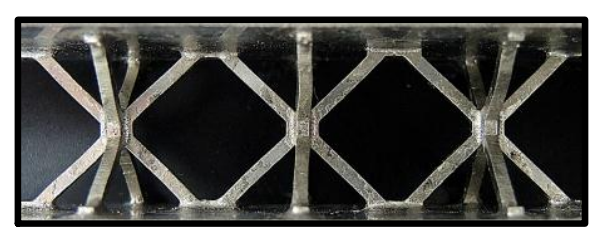

(a) $\varepsilon=0.05$

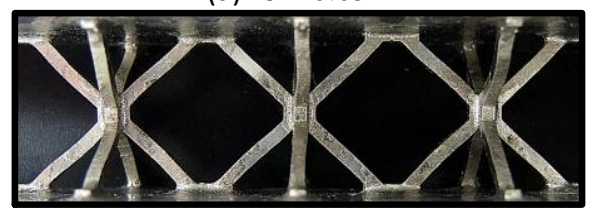

(b) $\varepsilon=0.07$

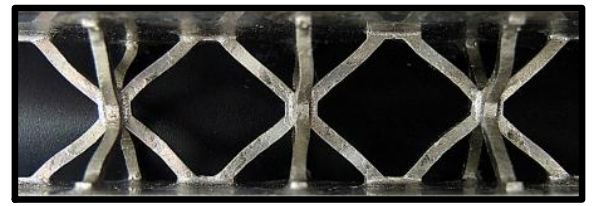

(c) $\quad \varepsilon=0.1$

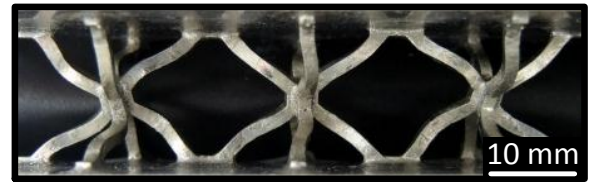

(d) $\varepsilon=0.2$

Fig. 7. Deformation photographs of the Hourglass truss lattice with $\bar{\rho}=2.71 \%$ during out-of-plane compression. $\varepsilon=0.07$ corresponds to the peak strength, and formation of a hinge in the middle of a truss member is seen in (b).

Fig. 7 shows the out-of-plane compressive deformation behaviors of the Hourglass lattice with $\bar{\rho}=2.71 \%$. Bending of the truss members, Fig. 7(a), begins to occur at loads prior to the attainment 
of the peak strength. Fig. 7 (b) corresponds to the peak strength, and formation of a hinge in the middle of a truss member can be observed. Softening coincides with both the formation of a plastic hinge near the middle of the truss members (Fig. 7(c)) and the following overall buckling of the lattice cell (Fig. 7(d)). Neither truss member fracture nor node failure is observed in any (pyramidal and Hourglass truss lattices) of the compression experiments performed.

\subsection{Comparison of predictions with measurements}

The expression for the out-of-plane compressive strength of the snap-fit octet-truss lattice structure has been discussed in detail by Dong et al. [12]. Similar to the snap-fit octet-truss lattice at high relative densities (low aspect ratio trusses), the out-of-plane compressive strength for an Hourglass truss lattice made from a rigid ideally plastic material is controlled by plastic yielding. The peak out-of-plane compressive strength, $\sigma_{33}^{\mathrm{pk}}$, in an Hourglass truss lattice or a pyramidal truss lattice failing by plastic yielding is then given by

$$
\sigma_{33}^{\mathrm{pk}}=\frac{2 \sqrt{2} t^{2}}{A} \cdot \sigma_{\mathrm{y}}
$$

where the unit cell cross-sectional area $A=(l+(b+c) / \sqrt{2})^{2}$ and $A=(\sqrt{2} l+(2 m+\sqrt{2} t+b+c) / 2)^{2}$ for the Hourglass truss lattice and the pyramidal truss lattice. In the limit of vanishing node size, the unit cell cross-sectional area $A$ can be simplified to $l^{2}$ and $2 l^{2}$, respectively, and Eq. (6) reduces to the peak out-of-plane compressive strength expression for the two ideal topology truss lattices, $\sigma_{33}^{\mathrm{pk}} \approx \bar{\rho} \sigma_{\mathrm{y}} / 2$.

If an Hourglass truss lattice is constructed from very slender trusses, the trusses collapse by elastic buckling under out-of-plane compression. The peak compressive strength in this case is found by replacing $\sigma_{\mathrm{y}}$ in Eq. (6) with the truss elastic bifurcation stress. 


$$
\sigma_{\mathrm{c}}=\frac{\pi^{2} k^{2} E_{\mathrm{s}}}{12} \cdot\left(\frac{t}{l}\right)^{2}
$$

where the factor $k$ depends on the rotational stiffness of the nodes: $k=1$ or $k=2$ for a pin-ended or built-in end conditions, respectively. In the experiments, no evidence of node rotation is observed when the peak strengths appear and we take $k=2$. In the limit of vanishing node size, combining Eqs. (3) and (4), Eq. (7) reduces to $\sigma_{\mathrm{c}} \approx 2 \cdot\left(\pi^{2} k^{2} E_{0} / 48 \sqrt{2}\right) \cdot \bar{\rho}$ for Hourglass lattices, and $\sigma_{\mathrm{c}} \approx\left(\pi^{2} k^{2} E_{\mathrm{s}} / 48 \sqrt{2}\right) \cdot \bar{\rho}$ for pyramidal lattices, respectively. From these simplified equations, it is clearly seen that the out-of-plane compressive strength of the Hourglass truss lattice is twice that of the pyramidal truss lattice with the same relative density, when their trusses collapse by elastic buckling at the same time.

If the truss material has a non-zero post yield strain hardening rate, the inelastic buckling strength

$$
\sigma_{\mathrm{c}}=\frac{\pi^{2} k^{2} E_{\mathrm{t}}}{12} \cdot\left(\frac{t}{l}\right)^{2}
$$

is defined by Shanley-Engesser tangent modulus theory [27-28]. In the limit of vanishing node size, comprising Eqs. (3) and (4), Eq. (8) reduces to $\sigma_{\mathrm{c}} \approx 2 \cdot\left(\pi^{2} k^{2} E_{\mathrm{t}} / 48 \sqrt{2}\right) \cdot \bar{\rho}$ for Hourglass lattices, and $\sigma_{\mathrm{c}} \approx\left(\pi^{2} k^{2} E_{\mathrm{t}} / 48 \sqrt{2}\right) \cdot \bar{\rho}$ for pyramidal lattices, respectively.

The out-of-plane compressive strengths $\sigma_{33}^{\mathrm{pk}}$ of both lattices are functions of the strut $(t / l)^{2}$ ratios, as well as strut material mechanical properties, as seen in Eqs. (6)-(8). Furthermore, $(t / l)^{2}$ scales with the relative density $\bar{\rho}$. Thus, the relative out-of-plane compressive strengths $\sigma_{33}^{\mathrm{pk}} / \sigma_{\mathrm{y}}$ are plotted against the relative density $\bar{\rho}$ of snap-fit lattices in Fig. 8 and 9. Relative density $\bar{\rho}$ takes into account the extra nodal volume (Eq. (1)) on all the figures. Fig. 8 shows both the tensile model and the compressive model (i.e. the analysis model based on the tensile and compressive 
stress-strain response) predictions of the Hourglass lattices, which are compared with the experimental data. We find that the inelastic buckling predicted curve by the compressive model changes abruptly at $\bar{\rho}=0.0173$ and become higher than its yielding predicted curve thereafter, since the parent material starts to strain harden and the inelastic bifurcation stress $\sigma_{\mathrm{c}}>\sigma_{\mathrm{y}}$. When $\bar{\rho}<0.0173$, including the range $\bar{\rho}<0.0005$ seen in the partial enlarged detail, the elastic buckling and inelastic buckling predictions of the tensile model are consistent with those of the compressive model. This results from almost the same strain-stress response of the two models before the parent material strain hardening (see Fig. 5). In this range both model predictions are in excellent agreement with the measurements. Nevertheless, the measurements are in good agreement with the compressive model but the theoretical values are evidently low as predicted by the tensile model when $\bar{\rho}>0.0173$. Since the strut is subjected to the compressive load, and the tangent modulus $E_{\mathrm{t}}$ of the compressive strain-stress response is evidently higher than the tensile one (see Fig. 5). Comparison of model results and measurements indicates that for the lattices with high relative density which strut alloy exhibits evident strain hardening, the out-of-plane compressive strength fails to be predicted by the tensile model, but is in excellent agreement with the compressive model. Therefore, as a more accurate model, compressive model is selected to predict the other compressive measurements in this paper.

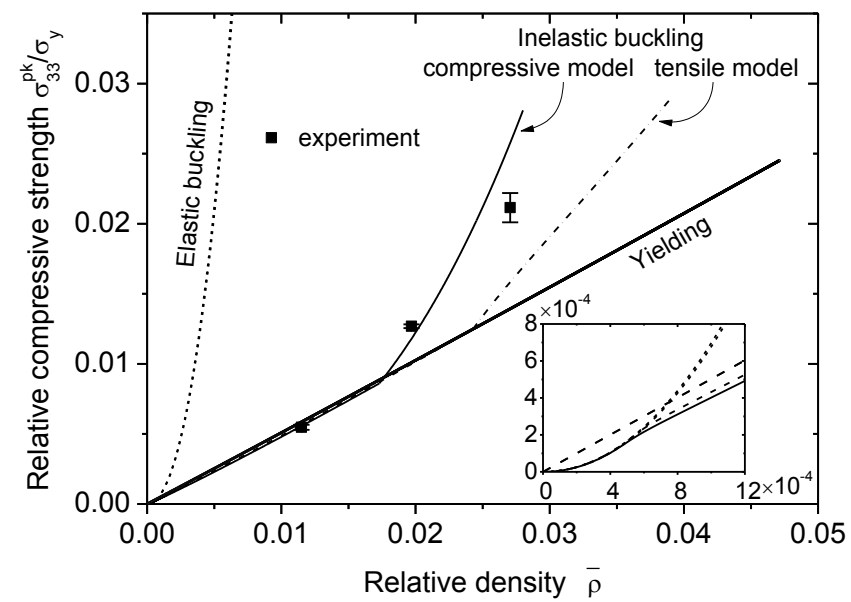


Fig. 8. Predicted and measured relative out-of-plane compressive strengths (normalized by the solid yield strength) plotted against relative density $\bar{\rho}$ of the snap-fit Hourglass truss lattice. Both the compressive model and tensile model predictions are shown. (Experimental error reported as \pm standard deviation).

For the snap-fit Hourglass lattice and the snap-fit pyramidal lattice, the measurements and compressive model predictions of the relative out-of-plane compressive strength plotted against relative density $\bar{\rho}$ are compared in Fig. 9. Table 3 also summarizes the predicted and experimental data. Measurements of both truss lattices are in good agreement with the inelastic buckling predictions of the compressive model. The discrepancies between experiments and predictions for lattice A, B, C, D, E, and F are $-4.11 \%, 6.71 \%,-18.93 \%,-20.96 \%,-0.24 \%, 5.95 \%$, respectively. Fig. 9(b) shows that the strength of the Hourglass lattice is higher than that of the pyramidal lattice when $\bar{\rho}<0.001$, a transition relative density from elastic to inelastic buckling of the pyramidal truss lattice. The former is about twice the latter when $\bar{\rho}>0.0005$. Since the $(t / l)^{2}$ ratio of the Hourglass lattice is about twice that of the pyramidal lattice at the same relative density (Eq. (1) and (2)). When $0.001<\bar{\rho}<0.0173$, both topology lattices exhibit low strain hardening, and the inelastic bifurcation stress $\sigma_{\mathrm{c}}$ approximately tends to a constant $\sigma_{\mathrm{y}}$. Thus the inelastic buckling predictions of the two topology lattices are close and both converge with their yielding predictions. When $\bar{\rho}>0.0173$, the Hourglass lattice material exhibits high strain hardening $\left(\sigma_{\mathrm{c}}>\sigma_{\mathrm{y}}\right)$, but the pyramidal lattice material still exhibits low strain hardening $\left(\sigma_{\mathrm{c}} \approx \sigma_{\mathrm{y}}\right)$. Therefore, the Hourglass truss lattices have higher inelastic buckling predictions and measurements in this range than the pyramidal truss lattices.

Comparison of the two lattice results indicates that the out-of-plane strength of the Hourglass truss lattice is evidently higher than that of the pyramidal truss lattice, unless the material of the two lattices exhibits low strain hardening at the same time. The increase of the out-of-plane compressive strength of the Hourglass truss lattice is a direct consequence of the increase of its strut slenderness ratio, $t / l$. 

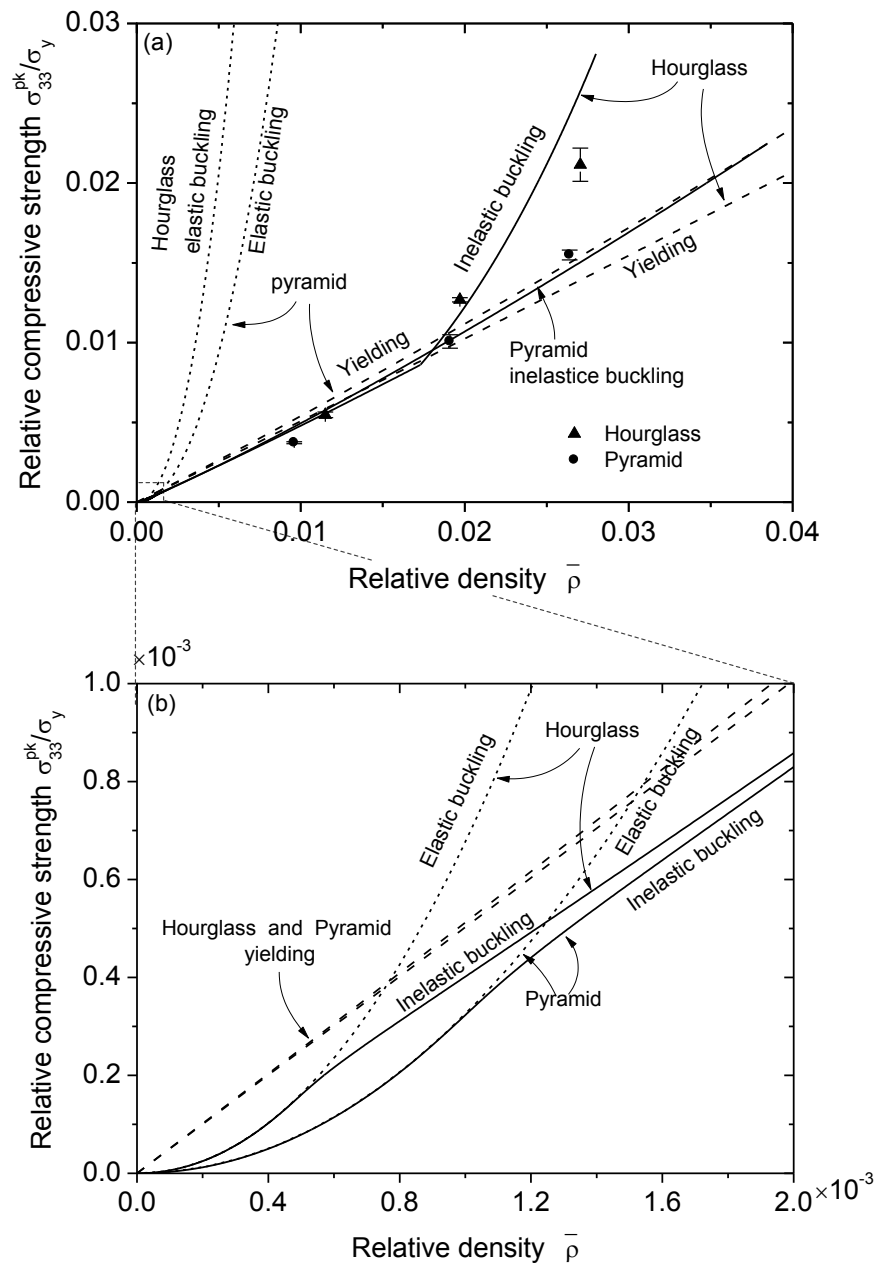

Fig. 9. Measurements and compressive model predictions of the relative out-of-plane compressive strength (normalized by the solid yield strength) plotted against relative density $\bar{\rho}$ of both the snap-fit Hourglass truss lattices and the snap-fit pyramidal truss lattices. The partially enlarged details of the compressive model predictions at low relative densities are shown in (b). (Experimental error reported as \pm standard deviation).

\subsection{Comparisons with competing truss lattice topologies}

The measured out-of-plane compressive strengths for periodic cellular metals with a variety of topologies are summarized in structural property charts of Fig. 10. All the samples are constructed from 304 stainless steel alloys of similar strength [9,29]. Fig. 10 shows that the normalized out-of-plane compressive strength coefficients $\Sigma=\sigma_{33}^{\mathrm{pk}} /\left(\sigma_{\mathrm{y}} \bar{\rho}\right)$ of the snap-fit Hourglass truss lattices in this paper are compared with competing stainless steel truss lattices, including the snap-fit pyramidal truss lattices in this paper, the pyramidal truss lattices made by folding perforated metal 
sheets and square honeycomb. The out-of-plane compressive strengths of Hourglass lattices are clearly superior to the other topologies at low relative density. Results indicate that the peak strengths of the Hourglass lattices are $26 \%-47 \%$ higher than those of the pyramidal lattices with similar relative densities. At a relative density of about $2.7 \%$, the normalized strength coefficient $\sigma_{33}^{\mathrm{pk}} /\left(\sigma_{\mathrm{y}} \bar{\rho}\right)$ of the Hourglass truss lattice is about $70 \%$ stronger than that of the pyramidal truss lattice based upon the perforating and folding method, and about $40 \%$ stronger than that of the square honeycomb. In addition, the snap-fit pyramidal lattices investigated here possess higher compressive strengths than the pyramidal lattices made by folding perforated sheets. This appears to be a consequence of more imperfections from the perforating and folding method, including truss waviness and small variations in the lengths of trusses created. Therefore, Hourglass truss lattices appear to be attractive alternatives to conventional pyramidal truss lattices together with honeycombs under out-of-plane compression.

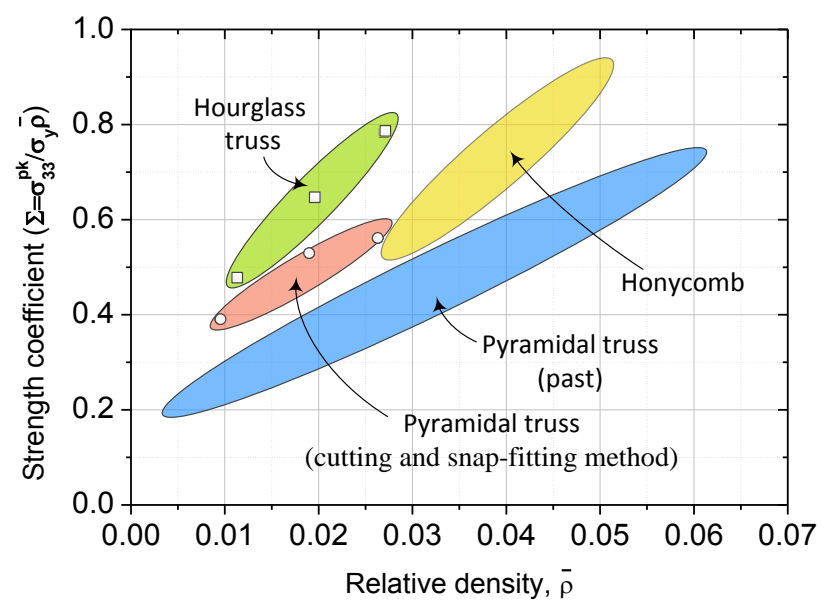

Fig. 10. Comparisons between the average peak strength coefficients, $\Sigma=\sigma_{33}^{\mathrm{pk}} /\left(\sigma_{\mathrm{y}} \bar{\rho}\right)$, of the Hourglass lattices, the pyramidal truss lattices made by snap-fit method, the pyramidal lattices made by folding perforated metal sheets and the square honeycomb as a function of relative density. They are all made from 304 stainless steel alloys of similar strength.

\section{In-plane compression}

Consider a lattice sandwich structure of length $L$ and width $B$ with clamped ends (constrained against rotation and displacement perpendicular to the structure) and subjected to an 
in-plane compressive load $P$, as sketched in Fig. 11(a). For all the in-plane compressive sandwich structures, the length $L=185 \mathrm{~mm}$ and $B=110 \mathrm{~mm}$. The sandwich structure comprises of face sheets of thickness $h_{\mathrm{f}}$ and either Hourglass lattice A or pyramidal lattice D (see Table 3) of height $H_{c}$, as seen in Fig. 11(b)-(d). Since the in-plane compressive responses of the both truss lattices are anisotropic, the response is dependent on the direction of compression. We define the direction of in-plane compression via the angle $\alpha$ with the $x_{1}$ axis, as shown in Fig. 4, such that $\alpha=0^{\circ}$ and $\alpha=90^{\circ}$ for applied in-plane compression loads, $P_{13}$ and $P_{23}$. Here, the in-plane displacements are applied in two principal directions of Hourglass sandwich structures: $\alpha=0^{\circ}$ and $\alpha=45^{\circ}$, and one direction of pyramidal sandwich structures: $\alpha=45^{\circ}$. Different sandwich structure geometries, specimen a to specimen e, are selected by varying the core type and the face sheet thickness, $h_{\mathrm{f}}$, as shown in Table 4.

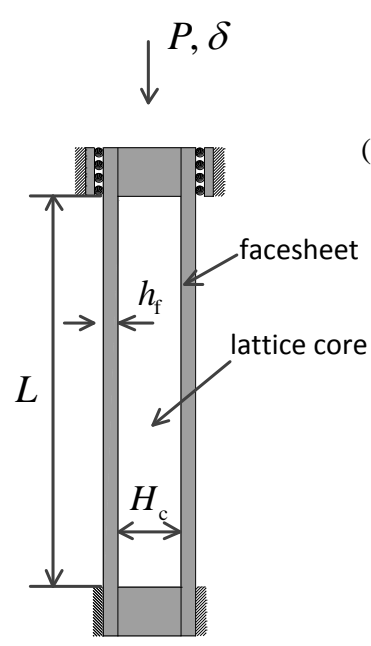

(a) (b)
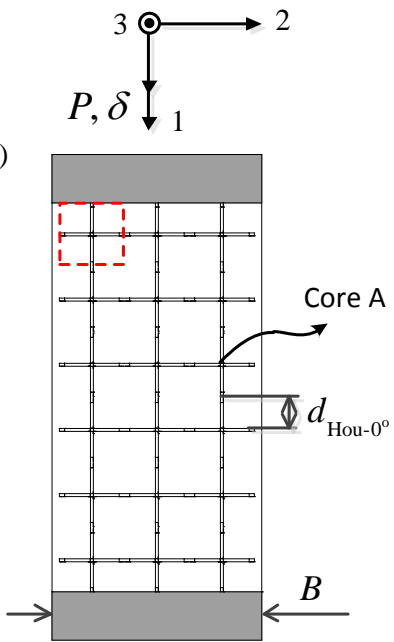

(b) (c)
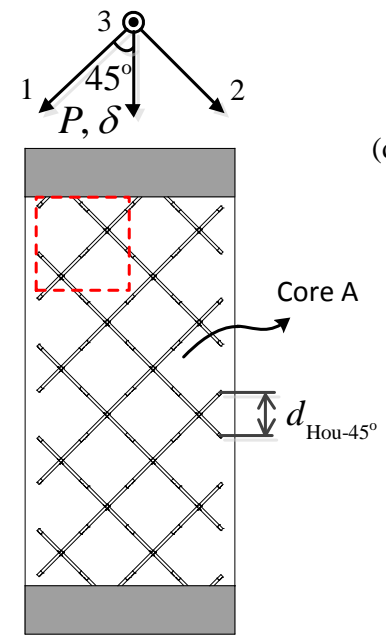

(c) (d)

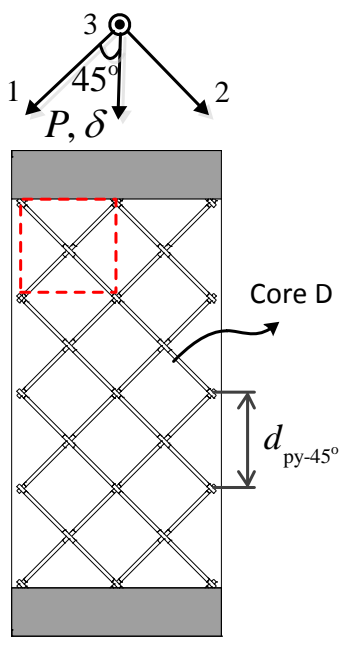

(d)

Fig. 11. Sandwich structures subjected to in-plane compression: (a) Schematic illustration of a sandwich structure under in-plane compression, (b) $\alpha=0^{\circ}$ Hourglass sandwich structure, (c) $\alpha=45^{\circ}$ Hourglass sandwich structure, (d) $\alpha=45^{\circ}$ pyramidal sandwich structure.

In-plane compression tests on the lattice sandwich structures are carried out in accordance with the ASTM standard C364-61 (1989) [30]. The in-plane compressive responses of the pyramidal lattice sandwich structures and the Hourglass lattice sandwich structures are measured at a nominal 
applied strain-rate of $10^{-4} \mathrm{~s}^{-1}$. In order to prevent the rotation of specimen ends, the specimens are filled at the ends with a two-component adhesive, providing a built-in loading condition, as sketched in Fig. 11. Care is taken to ensure that the lattice sandwich structures are flat and parallel. At least three repeated tests are conducted for each geometry structure structure in order to gauge the level of the experimental scatter.

Table 4

Summary of the sandwich structure design parameters in the in-plane compression tests along with the predicted and average measured peak loads .

\begin{tabular}{|c|c|c|c|c|c|}
\hline & \multicolumn{3}{|c|}{ Hourglass sandwich structure } & \multicolumn{2}{|c|}{ Pyramidal sandwich structure } \\
\hline & $\mathrm{a}$ & $\mathrm{b}$ & $\mathrm{c}$ & $\mathrm{d}$ & $\mathrm{e}$ \\
\hline Core type & $\mathrm{A}$ & A & $\mathrm{A}$ & $\mathrm{D}$ & $\mathrm{D}$ \\
\hline Load direction $(\alpha)$ & $0^{\circ}$ & $45^{\circ}$ & $0^{\circ}$ & $45^{\circ}$ & $45^{\circ}$ \\
\hline$h_{\mathrm{f}}(\mathrm{mm})$ & 0.47 & 0.47 & 0.9 & 0.47 & 0.9 \\
\hline Predicted $P_{\mathrm{pk}}(\mathrm{KN})$ & 16.04 & 8.92 & 36.16 & 2.47 & 17.21 \\
\hline Average of measured $P_{\mathrm{pk}} \quad(\mathrm{KN})$ & 11.28 & 7.02 & 36.22 & 3.22 & 19.11 \\
\hline Difference & $-29.70 \%$ & $-21.30 \%$ & $0.18 \%$ & $30.47 \%$ & $11.04 \%$ \\
\hline
\end{tabular}

At least four failure modes exist for Hourglass sandwich structures and pyramidal sandwich structures under in-plane compressive loading: (i) macro elastic buckling, (ii) macro inelastic buckling, (iii) local elastic face sheet buckling, and (iv) local inelastic face sheet buckling.

\subsection{In-plane compression response}

Fig. 12(a) and (b) shows the in-plane compressive responses of samples a to $\mathrm{e}$ and the corresponding deformation histories of samples $\mathrm{a}$ and $\mathrm{b}$ are presented in Fig. 12 (c) and (d), respectively. Most of the samples exhibited similar load-displacement responses including a region of nominally elastic response, peak load associated with buckling of the face sheets, and a progressive softening thereafter. Results show that the in-plane compression collapse loads of Hourglass samples a and b are 3.50 and 2.18 times that of pyramidal sample d, respectively. Moreover, the collapse load of Hourglass sample $\mathrm{c}$ is 1.90 times that of pyramidal sample e. 

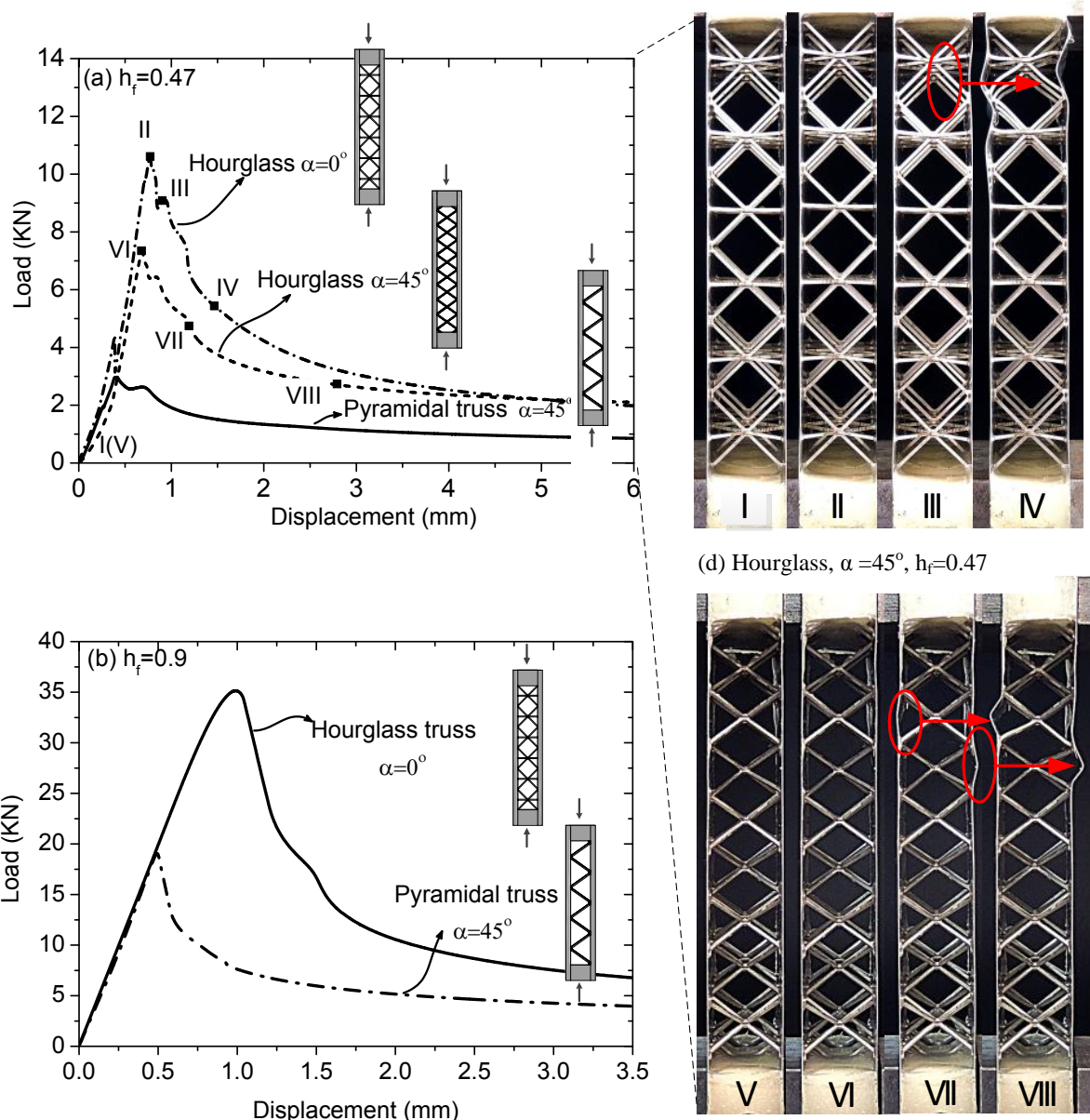

Fig. 12. In-plane compressive load/displacement responses for five lattice sandwich structures: (a) $0.47 \mathrm{~mm}$ thickness face sheets with Hourglass- $0^{\circ}$, Hourglass- $45^{\circ}$ and pyramidal lattice sandwich structures (i.e. samples a, b, and d), (b) $0.9 \mathrm{~mm}$ thickness face sheets with Hourglass- $0^{\circ}$ and pyramidal sandwich structures (i.e. samples $\mathrm{c}$ and e). The deformation history of the lattice structures: (c) sample a and (d) sample b.

\subsection{Comparison of predictions with measurements}

For the pyramidal sandwich structure, approximate analytical expressions for the in-plane compressive collapse load, $P$, can be developed following [15] and are summarized by the equations listed in Table 5. The in-plane compressive collapse loads do not depend upon the lattice topology but depend upon the inter-node spacing, $d$, under the local face buckling failure mode. Therefore, the analytical expressions can also characterize the in-plane compressive collapse loads of Hourglass lattice sandwich structures. However, since the inter-node spacing $d$ of the two lattice structures differ obviously, the predicted results by the analytical expressions are different. 
The inter-node spacing $d$ of $\alpha=0^{\circ}$ Hourglass sandwich structure, $\alpha=45^{\circ}$ Hourglass sandwich structure and $\alpha=45^{\circ}$ pyramidal sandwich structure are about $H_{c} / 2, \sqrt{2} H_{c} / 2$ and $\sqrt{2} H_{c}$, respectively, as seen from Fig. 11. In these lattice sandwich structures, we set $k^{\prime}=1$ for the face sheet buckling as pin-jointed struts. For Hourglass sandwich structures and pyramidal sandwich structures, we obtain the shear rigidity, $\mathrm{S}$, as

$$
\begin{gathered}
S=G_{c} B H_{\mathrm{c}} \\
G_{\mathrm{c}}=\frac{2 t^{2}}{A} \sin \omega \cos ^{2} \omega \cdot E_{\mathrm{s}}
\end{gathered}
$$

Table 5

Analytical expressions for the in-plane compression collapse loads of Hourglass sandwich structures and pyramidal sandwich structures.

In-plane compressive failure mode

Analytical collapse load expression

Macro elastic buckling

$$
P_{\mathrm{MEB}}=\frac{\frac{2 k^{4} \pi^{4} D_{\mathrm{f}} D_{0}}{L^{4}}+\frac{k^{2} \pi^{2} D}{L^{2}} S}{\frac{k^{2} \pi^{2} D_{0}}{L^{2}}+S} \text {, when } P_{\mathrm{MEB}}<\frac{4 B h_{\mathrm{f}} \sigma_{\mathrm{y}}}{\sqrt{3}}
$$

Macro inelastic buckling

$$
P_{\mathrm{MIB}}=\frac{\frac{2 k^{4} \pi^{4} D_{\mathrm{f}} D_{0}}{L^{4}}+\frac{k^{2} \pi^{2} D}{L^{2}} S}{\frac{k^{2} \pi^{2} D_{0}}{L^{2}}+S} \text {, when } P_{\mathrm{MEB}} \geq \frac{4 B h_{\mathrm{f}} \sigma_{\mathrm{y}}}{\sqrt{3}}
$$

Local elastic face buckling

$$
P_{\mathrm{EFB}}=\frac{k^{\prime 2} \pi^{2} E_{\mathrm{s}} B}{6\left(1-v^{2}\right)} \frac{h_{\mathrm{f}}{ }^{3}}{d^{2}} \text {, when } \frac{h_{\mathrm{f}}}{d}<\sqrt{\frac{24\left(1-v^{2}\right) \sigma_{\mathrm{y}}}{\sqrt{3} k^{\prime 2} \pi^{2} E_{\mathrm{s}}}}
$$

Local inelastic face buckling

$$
P_{\mathrm{IFB}}=\frac{k^{\prime 2} \pi^{2} E_{\mathrm{t}} B}{6} \frac{h_{\mathrm{f}}{ }^{3}}{d^{2}}, \text { when } \frac{h_{\mathrm{f}}}{d} \geq \sqrt{\frac{24\left(1-v^{2}\right) \sigma_{\mathrm{y}}}{\sqrt{3} k^{\prime 2} \pi^{2} E_{\mathrm{s}}}}
$$

Here $D=2 D_{\mathrm{f}}+D_{0}$; when $P_{\mathrm{MEB}}<\frac{4 B h_{\mathrm{f}} \sigma_{\mathrm{y}}}{\sqrt{3}}, D_{0}=\frac{E}{1-v^{2}} \frac{B h_{\mathrm{f}}\left(h_{\mathrm{f}}+H_{c}\right)^{2}}{2}$ and $D_{\mathrm{f}}=\frac{E_{\mathrm{s}}}{1-v^{2}} \frac{B h_{\mathrm{f}}^{3}}{12} ;$ when $P_{\mathrm{MEB}} \geq \frac{4 B h_{\mathrm{f}} \sigma_{\mathrm{y}}}{\sqrt{3}}$, $D_{0}=\frac{E_{\mathrm{t}} B h_{\mathrm{f}}\left(h_{\mathrm{f}}+H_{c}\right)^{2}}{2}$ and $D_{\mathrm{f}}=\frac{E_{\mathrm{t}} B h_{\mathrm{f}}^{3}}{12}$. For the structures in this paper, $k=2, k^{\prime}=1$ and $v=0.3$.

The regimes of dominance of the failure modes described above can be illustrated in a collapse mechanism map. In constructing such a map, it is assumed that the collapse mode is the one associated with the lowest collapse load $P$. These maps are developed as a function of non-dimensional parameters $h_{\mathrm{f}} / H_{c}$ and $L / H_{c}$. The boundaries of each failure mode are obtained 
by evaluating the minimum normalized collapse load $P /\left(\sigma_{\mathrm{y}} B H_{c}\right)$ at given values of $h_{\mathrm{f}} / H_{c}$ and $L / H_{c}$. Failure mechanism maps for the three lattices (ie. $\alpha=0^{\circ}$ core A, $\alpha=45^{\circ}$ core A and $\alpha=45^{\circ}$ core D) shown in Fig. 11(b) to (d), which are all constructed in one figure and compared, as seen in Fig. 13. The mechanism map is divided into a total of nine areas: I-IX, by the boundaries. Area IV contains the five unlabeled areas. Simultaneously, each group of boundary lines of the three lattices divides the map into four regimes, dominated respectively by the four failure modes described above. The regimes where the three lattice sandwich structures possess the same failure modes are pointed out in Fig. 13, i.e. regimes of I, III, VIII and IX, and these four regimes corresponds to the local elastic face buckling mode, the local inelastic face buckling mode, the macro elastic buckling mode and the macro inelastic buckling mode, respectively.

Combining expressions in Table 5, the comparison results of the three topology lattice sandwich structures are as follows. In regimes I-IV, the collapse load of $\alpha=0^{\circ}$ Hourglass sandwich structure is higher than that of $\alpha=45^{\circ}$ Hourglass sandwich structure, and the pyramidal sandwich structure the lowest. In areas V-VII, the peak load of $\alpha=45^{\circ}$ Hourglass sandwich structure is equal to that of $\alpha=0^{\circ}$ Hourglass sandwich structure, and the pyramidal sandwich structure the lowest. In areas VIII and IX, all the collapse loads of the three topology sandwich structures are approximately equal. In summary, in the entire shadow area (both the yellow and the gray shadow), Hourglass sandwich structure of $\alpha=0^{\circ}$ or $\alpha=45^{\circ}$ provides higher peak collapse loads to $\alpha=45^{\circ}$ pyramidal lattice sandwich structure. Hence, the Hourglass lattice sandwich structure appears theoretically to be an attractive alternative to conventional pyramidal lattice under in-plane compression, and this is because of the smaller inter-node spacing, $d$, of the pyramidal lattice sandwich structure. Moreover, it is noted that tested geometries are selected in an attempt to exhibit the local face sheet buckling failure modes, and marked in the mechanism map, where $P_{\mathrm{py}-45^{0}}<P_{\mathrm{Hou}-45^{0}}<P_{\mathrm{Hou}-0^{0}}$ in theory. 


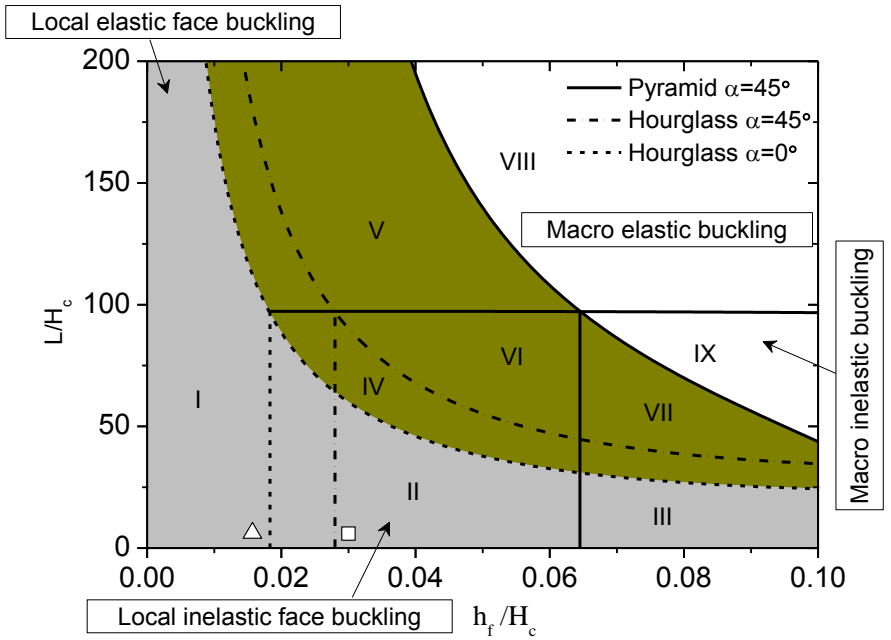

Fig. 13. Collapse mechanism map of $\alpha=45^{\circ}$ pyramidal sandwich structure with core $\mathrm{D}, \alpha=0^{\circ}$ Hourglass sandwich structure and $\alpha=45^{\circ}$ Hourglass sandwich structure with core A. The geometries tested here are indicated on the map.

The relative in-plane compressive local face buckling loads $\overline{P_{\mathrm{FB}}}=P_{\mathrm{FB}} /\left(B H_{c} \sigma_{\mathrm{y}}\right)$ of sandwich structures are functions of the ratio $h_{\mathrm{f}} / H_{c}$ with a given cell width $d$, as seen in Table 5. For the three kinds of lattice structures, the compressive model predictions and measurements of the relative in-plane compressive local face buckling loads $\overline{P_{\mathrm{FB}}}$ are then plotted against the ratio $h_{\mathrm{f}} / H_{c}$ in Fig. 14. Note that the relations in Fig. 14 are applicable for the lattice structure sizes in the gray shadow region of Fig. 13, where the collapse modes of the three kinds of lattice sandwich structures are all local face sheet buckling. Table 4 also summarizes the predicted and experimental values. In some cases the measured loads are significantly lower than the predictions. Not only because there are imperfections in the manufactured sample, but because the sample lies at the boundary of the local elastic and local inelastic face buckling modes and are expected to display a strong sensitivity to initial imperfections [31-35]. Such discrepancies between predictions and measurements are not uncommon in structures made from stainless steel which has a sharp transition from its elastic response (Young's modulus $E_{\mathrm{s}}=213 \mathrm{GPa}$ ) to a linearly hardening plastic response with a tangent modulus $E_{\mathrm{t}} \approx 2 \mathrm{GPa}[15]$. The measured failure load of sample $\mathrm{d}$ is higher than the predicted value. Such a discrepancy between the prediction and the measurement is a consequence 
that the nodes between the facesheets and the core lead to the decrease of the inter-node spacing in measurements.

Examination of Fig. 14 shows that the transition $h_{\mathrm{f}} / H_{c}$ ratios from facesheet elastic buckling to inelastic buckling of $\alpha=0^{\circ}$ Hourglass lattice sandwich structure, $\alpha=45^{\circ}$ Hourglass lattice sandwich structure and $\alpha=45^{\circ}$ pyramidal lattice sandwich structure are $\sim 0.018, \sim 0.025$ and $\sim 0.050$, respectively, which is consistent with Fig 13 . When $h_{\mathrm{f}} / H_{c}<0.018$, the relative in-plane compressive loads of $\alpha=0^{\circ}$ and $\alpha=45^{\circ}$ Hourglass sandwich structures are almost 8 times and 4 times that of $\alpha=45^{\circ}$ pyramidal sandwich structure, respectively. Local elastic face buckling is predicted and observed in samples a, b and $\mathrm{d}$. When $0.018<h_{\mathrm{f}} / H_{c}<0.025$, the relative in-plane compressive load of $\alpha=0^{\circ}$ Hourglass lattice sandwich structure becomes close to that of $\alpha=45^{\circ}$ Hourglass lattice sandwich structure, and is still almost 4 times that of $\alpha=45^{\circ}$ pyramidal lattice sandwich structure. When $0.025<h_{\mathrm{f}} / H_{c}<0.050$, the relative in-plane compressive load of $\alpha=0^{\circ}$ Hourglass sandwich structure converges to that of $\alpha=45^{\circ}$ Hourglass sandwich structure, and they are both higher than that of $\alpha=45^{\circ}$ pyramidal sandwich structure. Local inelastic face buckling failure is predicted and observed in samples $c$ and e. Since the facesheet alloy exhibits low strain hardening, the relative in-plane compressive load of $\alpha=45^{\circ}$ pyramidal sandwich structure begins to converge to that of $\alpha=0^{\circ}$ and $\alpha=45^{\circ}$ Hourglass lattice sandwich structures when $h_{\mathrm{f}} / H_{c}>0.050$. Comparison of results similar to the out-of-plane compressive strengths is that, unless the facesheet alloy of the three lattice sandwich structures exhibits low strain hardening at the same time (when $h_{\mathrm{f}} / H_{c}>0.04$ ), the in-plane collapse load of the Hourglass lattice sandwich structure is evidently higher than that of the pyramidal lattice sandwich structure. The decrease of the inter-node spacing, $d$, leads to the increase of the in-plane compressive load of the Hourglass lattice sandwich structure. 


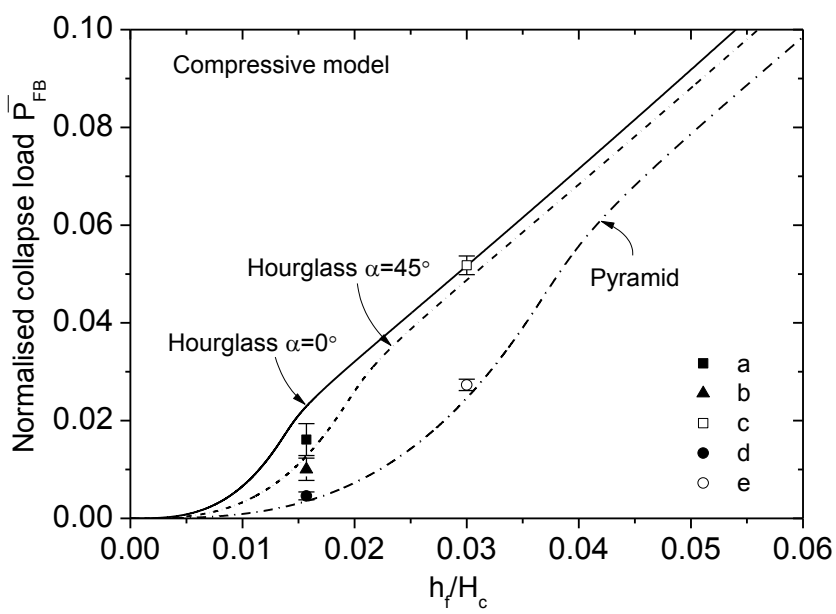

Fig. 14. Measurements and compressive model predictions of the relative in-plane compressive local face buckling loads (normalized by $\sigma_{\mathrm{y}} B H_{c}$ ) plotted against $h_{\mathrm{f}} / H_{c}$ of the $\alpha=0^{\circ}$ Hourglass sandwich structure, $\alpha=45^{\circ}$

Hourglass sandwich structure and $\alpha=45^{\circ}$ pyramidal sandwich structure. (Experimental error reported as \pm standard deviation).

\section{Conclusion}

In this paper, an Hourglass truss lattice sandwich structure is developed and manufactured via a snap-fit and vacuum brazing approach. The compressive mechanical properties of Hourglass and pyramidal lattice sandwich structures have been investigated theoretically and experimentally. In theory, the compressive model, proving to be more accurate than the tensile model, is proposed to predict the compressive strengths. The measured collapse loads are in reasonable agreement with the predictions in most cases. In the experiments, the measured out-of-plane compressive strengths of the Hourglass lattices (relative densities from $1.15 \%$ to $2.71 \%$ ) improve by $26 \%-47 \%$ compared with the snap-fit pyramidal lattices. The out-of-plane compressive strengths of the Hourglass lattices also outperform some other existing topologies. Meanwhile, the measured in-plane compressive collapse loads of Hourglass lattice sandwich structures are up to 3.5 times (the maximum time) that of pyramidal lattice sandwich structures with the same structure sizes. In all, this research designs and prepares a new Hourglass lattice sandwich structure possessing high peak out-of-plane compressive strength, high in-plane compressive collapse load, and simple fabricating process. 


\section{Acknowledgements}

The present work was supported by the National Science Foundation of China under Grant No. 11432004.

\section{References}

[1] M.F. Ashby, A.G. Evans, N.A. Fleck, L.J. Gibson, Hutchinson, J.W., Wadley, H.N.G., Metal foams: A Design Guide, Butterworth Heinemann, London, 2000.

[2] F.W. Zok, S.A. Waltner, Z. Wei, H.J. Rathbun, McMeeking, R.M., Evans, A.G., A protocol for characterizing the structural performance of metallic sandwich panels: application to pyramidal truss cores, Int. J. Solids Struct. 41(2004) $6249-6271$.

[3] D.T. Queheillalt, H.N.G. Wadley, Titanium alloy lattice truss structures, Mater. Des. 30(2009) 1966-75.

[4] J.Y. Liu, X. Zhu, T.Y. Li, Z.G. Zhou, L.Z. Wu, L. Ma, Experimental study on the low velocity impact responses of all-composite pyramidal truss core sandwich panel after high temperature exposure, Compos. Struct. 116 (2014) $670-81$.

[5] H.J. Rathbun, Z. Wei, M.Y. He, F.W. Zok, A.G. Evans, D.J. Sypeck, H.N.G. Wadley, Measurement and simulation of the performance of a lightweight metallic sandwich structure with a tetrahedral truss core, J. Appl. Mech. Trans. ASME 71 (3) (2004) 368-374.

[6] J. Wang, A.G. Evans, K. Dharmasena, H.N.G. Wadley, On the performance of truss panels with Kagomé cores, Int. J. Solids Struct. 40 (2003), 6981-6988.

[7] J.H. Lim, K.J. Kang, Mechanical behavior of sandwich panels with tetrahedral and Kagomé truss cores fabricated from wires, Int. J. Solids Struct. 43 (2006) 5228-5246.

[8] Y.H. Lee, B.K. Lee, I. Jeon, K.J. Kang, Wire-woven bulk Kagome truss cores, Acta Mater. 55 (2007) 6084-6094.

[9] D.T. Queheillalt, H.N.G. Wadley, Cellular metal lattices with hollow trusses, Acta Mater. 53 (2005) 303-313.

[10] S. Yin, L.Z. Wu, S.R. Nutt, Compressive efficiency of stretch-stretch-hybrid hierarchical composite lattice cores, Mater. Des. 56(2014) 731-9.

[11] Q.C. Zhang, Y.J. Han, C.Q. Chen, T.J. Lu, Ultralight X-type lattice sandwich structure (I): Concept, fabrication and experimental characterization, Sci. China. Ser. E 52(9) (2009) 2670-80. 
[12] L. Dong, V.S. Deshpande, H.N.G. Wadley, Mechanical response of Ti-6Al-4V octet-truss lattice structures, Int. J. Solids Struct. 60-61(2015) 107-124.

[13] V.S. Deshpande, N.A. Fleck, Collapse of truss core sandwich beams in 3-point bending, Int. J. Solids Struct. 38 (2001) 6275-6305.

[14] S. Chiras, D.R. Mumm, A.G. Evans, N. Wicks, J.W. Hutchinson, K.P. Dharmasena, H.N.G. Wadley, S. Fichterd, The structural performance of near-optimized truss core panels, Int. J. Solids Struct. 39 (2002) 4093-4115.

[15] F. Cote, R. Biagi, H. Bart-Smith, V.S. Deshpande, Structural response of pyramidal core sandwich columns, Int. J. Solids Struct. 44 (2007) 3533-3556.

[16] I. Sridhar, N.A. Fleck, End compression of sandwich columns, Compos. Part A Appl. Sci. Manuf. 33 (3) (2002) $353-359$.

[17] B. Wang, L.Z. Wu, L. Ma, Y.G. Sun, S.Y. Du, Mechanical behavior of the sandwich structures with carbon fiber-reinforced pyramidal lattice truss core, Mater. Des. 31 (2010) 2659-2663.

[18] N. Wicks, J.W. Hutchinson, Optimal truss plates, Int. J. Solids Struct. 38 (2001) 5165-5183.

[19] F.K. Zok, H.J. Rathbun, Z. Wei, A.G. Evans, Design of metallic textile core sandwich panels, Int. J. Solids Struct. 40 (2003) 5707-5722.

[20] P. Moongkhamklang, D.M. Elzey, H.N.G. Wadley, Titanium matrix composite lattice structures, Compos. Part A Appl. Sci. Manuf. 39 (2008) 176-187.

[21] C. Zschernack, M.A. Wadee, C. Völlmecke, Nonlinear buckling of fibre-reinforced unit cells of lattice materials, Compos. Struct. 136(2016) 217-228.

[22] K. Finnegan, G. Kooistra, H.N.G. Wadley, V.S. Deshpande, The compressive response of carbon fiber composite pyramidal truss sandwich cores, Int. J. Mater. Res. 98 (2007) 1264-1272.

[23] G.Q. Zhang, B. Wang, L. Ma, J. Xiong, L.Z. Wu, Response of sandwich structures with pyramidal truss cores under the compression and impact loading, Compos. Struct. 100 (2013) 451-463.

[24] G.Q. Zhang, B. Wang, L. Ma, J. Xiong, J.S. Yang, L.Z. Wu, The residual compressive strength of impact-damaged sandwich structures with pyramidal truss cores, Compos. Struct. 105 (2013) 188-198.

[25] J. Xiong, R. Ghosh, L. Ma, A. Vaziri, Y.L. Wang, L.Z. Wu, Sandwich-walled cylindrical shells with lightweight metallic lattice truss cores and carbon fiber-reinforced composite face sheets, Compos. Part A Appl. Sci. Manuf. 56 (2014) 226-238.

[26] K.J.R. Rasmussen, Full-range stress-strain curves for stainless steel alloys, J. Constr. Steel Res. 59 (2003) 47-61. 
[27] S.P. Timoshenko, J.M. Gere, Theory of elastic stability, McGraw-Hill International Book Company, New York, 1985.

[28] F.R. Shanley, Mechanics of materials, McGraw-Hill, New York, 1967.

[29] M. Zupan, V.S. Deshpande, N.A. Fleck, The out-of-plane compressive behavior of woven-core sandwich plates, Euro. J. Mech. A/Solids. 23 (2004) 411-421.

[30] ASTM C364-61, Standard test method for edgewise compressive strength of flat sandwich constructions, American Society of Testing and Materials, 1989.

[31] F. Cote, V.S. Deshpande, N.A. Fleck, A.G. Evans, The out-of-plane compressive behavior of metallic honeycombs, Mat. Sci. Eng. A 380 (2004) 272-280.

[32] J.W. Hutchinson, Plastic buckling, Advances in Applied Mechanics, vol.14. Academic Press, New York, 1974.

[33] J.M.T. Thompson, G.W. Hunt, A general theory of elastic stability, Publisher: John Wiley and Sons, 1973.

[34] M.A. Wadee, A. Blackmore, Delamination from localized instabilities in compression sandwich panels, J. Mech. Phys. Solids. 49(2001) 1281-1299.

[35] M.A. Wadee, Localized buckling in sandwich struts with pre-existing delaminations and geometrical imperfections, J. Mech. Phys. Solids. 50(2002) 1767-1787. 


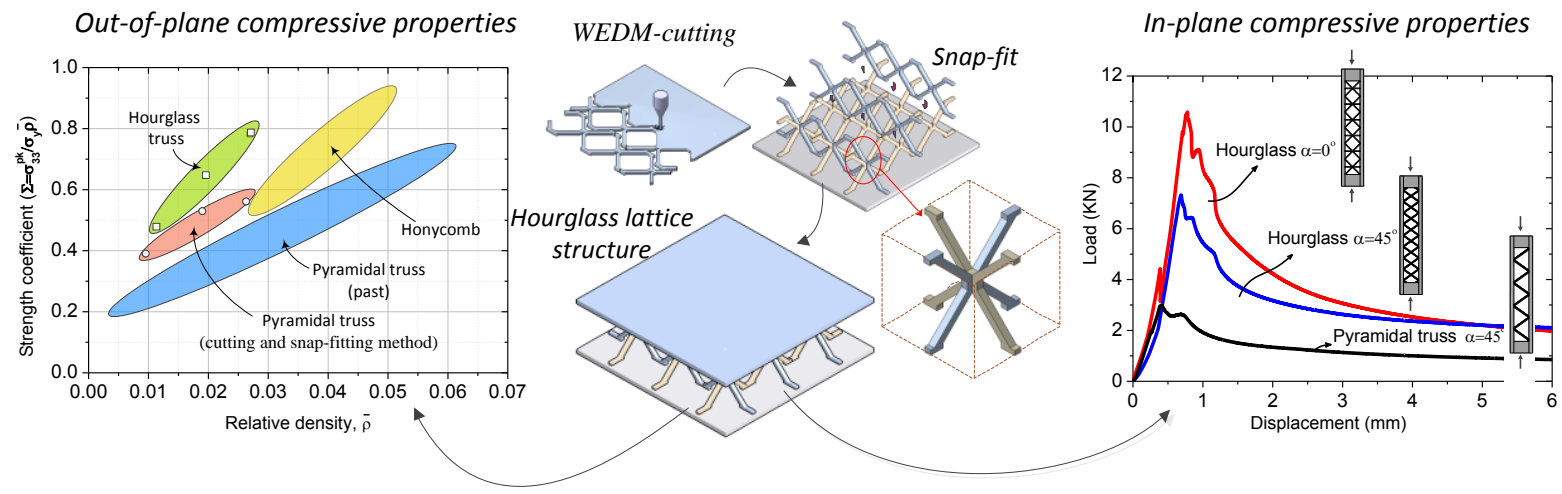

